\title{
A high corticosterone/DHEA-s ratio in young rats infected with Trypanosoma cruzi is associated with increased susceptibility
}

\author{
Ana Rosa Pérez ${ }^{1,2}$, Aymará Ana Bertoya1', Silvia Revelli1, Fabiana García ${ }^{1,2}{ }^{+}$ \\ 'Instituto de Inmunología, Facultad de Ciencias Médicas de Rosario, Universidad Nacional de Rosario, Rosario, Argentina \\ ${ }^{2}$ Consejo Nacional de Investigaciones Científicas y Técnicas, Buenos Aires, Argentina
}

\begin{abstract}
We have previously established that young male rats are more susceptible to the effects of Trypanosoma cruzi infection than adult rats. To explore underlying age-associated differences in disease outcome, we simultaneously assessed hormone levels and cytokine release throughout the acute infection period in young and adult rats infected with T. cruzi. Young rats were inoculated with $1 \times 10^{6}$ and adult rats with $7 \times 10^{6}$ blood trypomastigotes, according to their relative body weight. At zero, seven, 14, 21 and 28 days after infection, blood was collected for the determination of gonadal and adrenal hormones, tumor necrosis factor $\alpha$ (TNF- $\alpha$ ), interleukin (IL)-10 and specific IgM and IgG subtypes. Young animals displayed significantly higher parasitaemia values and an endocrine pattern that was characterised by elevated values in corticosterone (CT) and the CT/dehydroepiandrosterone-sulfate ratio, which favours immunosuppression and susceptibility. In contrast, adult male rats were able to restrict the parasite burden, which likely resulted from increased IgG antibody synthesis and oestradiol levels. Adult rats also showed a reduced TNF- $\alpha / I L-10$ ratio and less tissue damage. We conclude that young animals exhibited increased vulnerability to T. cruzi infection compared with adults and this is associated with an unsuitable immunoendocrine milieu.
\end{abstract}

Key words: immunoendocrine interactions - Chagas disease - adrenal cortex hormones

Chagas disease, which shows three successive phases (i.e., acute, intermediate and chronic), is caused by the protozoan parasite Trypanosoma cruzi and affects nearly 12 million people in South and Central American countries (WHO/PAHO 2007).

In humans, T. cruzi infection can occur at any age; however, a significant number of cases are detected before the age of 14 years and the highest frequency of cases are detected before the age of five (Storino \& Milei 1994). To obtain some insight into the age-associated differences during the course of the disease, our laboratory developed an experimental model in rats where inoculation with $T$. cruzi during weaning resulted in acute infection, which was characterised by high parasitaemia levels and inappropriate antibody production. In contrast, adult animals inoculated with T. cruzi only showed a mild disease with extremely low parasitaemia values (Revelli et al. 1987, Pascutti et al. 2003). These age-related susceptibility differences might be explained by immune system maturity and endocrine influences as both play an integral role in biological defence mechanisms (Roberts et al. 2001).

Recent experimental evidence from studies in adult mice and rats showed that there was an endocrine response associated with the immune process that strongly affected the course of $T$. cruzi infection and appeared

Financial support: SeCyT-UNR (MED 119, MED 256), Josefina Prats Foundation

SR an FG contributed equally to this work.

+ Corresponding author: fgarcia@fmedic.unr.edu.ar

Received 25 October 2010

Accepted 20 April 2011 to be influenced by basal levels of glucocorticoids, the kinetics of corticosterone (CT) release and gonadal axis manipulations (Corrêa-de-Santana et al. 2006, Roggero et al. 2006, do Prado et al. 1999).

Studies have also demonstrated that gender and age are important factors in determining the course and consequence of other parasite infections (e.g., schistosomiasis). Correlations were found between age, sex, the degree of morbidity and pathology; cytokines emerged as the possible mediators for these correlations (MoralesMontor \& Hall 2007). Interestingly, studies in adult animals with schistosomiasis showed that high testosterone levels diminished worm burden (Nakazawa et al. 1997), whereas reports from T. cruzi infected adult rats and mice exhibited opposite results (do Prado et al. 1999, del Vecchio Filipin et al. 2010).

Many studies have suggested that the strong interaction between the immune and neuroendocrine systems in coordinating responses during infectious processes is a generalised and adaptive defence reaction. This reaction seems to be characterised by inflammatory mediators, which are able to exert marked effects on the neuroendocrine system which in turn affects cytokine release. Moreover, an inadequate immunoendocrine response would fail to eradicate pathogens that cause sickness, including parasites (Besedovsky \& del Rey 1996, Pérez et al. 2009a, Roggero et al. 2009). Consequently, the present study aimed to analyse the age-dependent trends in the physiological levels of hormones and immune mediators during the acute period of infection in an experimental rodent model. We studied gonadal and adrenal hormone patterns, cytokines involved in susceptibility or resistance to T. cruzi [e.g., tumor necrosis factor $\alpha$ (TNF- $\alpha$ ) and interleukin (IL)-10] and specific antibodies (e.g., IgM, IgG and their subtypes). We also investigated cor- 
relations between endocrine and immunological parameters that could explain the enhanced susceptibility to $T$. cruzi infection that was observed in young rats compared with adult rats. The present study is the first to show that the age-related severity of T. cruzi infections is linked to hormonal modifications during the T. cruzi acute phase.

\section{SUBJECTS, MATERIALS AND METHODS}

Animals and parasites - Adult ( 80 days old) and young (21-28 days old) inbred male "l" rats were obtained by breeding in the animal facility at the School of Medicine, National University of Rosario (Argentina) and maintained according to the International Guidelines for the Care and Use of Laboratory Animals. The experimental protocol was approved by the Research and Bioethical Committees from the University. All rats were housed in groups of four per cage, maintained in a temperature controlled environment under a constant 12-h light-dark cycle and provided with a standard diet and water ad libitum. Rats were kept under routine laboratory conditions before experiments and they were allowed to acclimate for one week in the animal holding facility before infection. Infective blood trypomastigotes of the Tulahuén strain of $T$. cruzi were maintained by serial passage in CBi mice.

Experimental protocol - Rats were subcutaneously inoculated before puberty (21 days old) or during adulthood (70 days old) with $1 \times 10^{6}$ or $7 \times 10^{6}$ parasites in $0.1 \mathrm{~mL}$ saline solution, respectively, according to body weight (Pascutti et al. 2003). Control groups were only injected with saline solution. Rats were euthanized before infection (day 0 ) and seven, 14, 21 and 28 days post-infection (pi). To avoid stress, rats were handled daily by the same operators until sacrifice. Rats with piloerection or changes in body weight or behaviour (e.g., aggressiveness) were discarded. Rats were killed by decapitation in an adjacent laboratory 1-2 min after being taken from the animal house. Blood samples were always obtained between 8:00-10:00 am. for the determination of serum hormones and cytokines and the detection of specific antibodies. Bloodstream forms of $T$. cruzi were assessed under standardised conditions by direct microscopic observation of $5 \mu \mathrm{L}$ of heparinised tail vein blood at each time point. Data were expressed as number of parasites $/ \mathrm{mL}$. Tissues were removed to determine their weight, and histopathological studies were performed to evaluate tissue parasitism and inflammatory infiltrate. Each organ weight was assessed relative to body weight (relative weight).

Histopathology - Heart tissue, testes, epididymides, seminal vesicles and adrenal glands were removed at each time point and fixed in buffered formalin or Bouin for histopathology. Analysis of heart tissues was performed on five paraffin-embedded 5- $\mu \mathrm{m}$ sections from each rat. The sections were stained with haematoxylin and eosin for the evaluation of inflammatory foci, tissue parasitism, myocarditis and fibrosis. A similar histopathological procedure was performed with the other tissues. Foci of myocarditis were classified as previously described (Pérez et al. 2009b). Briefly, mild foci were determined as slight infiltrations with damage of one or two myocardial fibres, moderate-sized foci were defined as aggregated infiltrates compromising 2-5 muscle fibres and intense foci were defined as heavy accumulations of mononuclear cells with destruction of more than five muscle fibres. Sections from each organ were examined by an experienced pathologist blinded to the study groups.

Steroid hormone assay - Blood was collected in glass tubes and centrifuged at $1.500 \mathrm{rpm}$ for $10 \mathrm{~min}$ at room temperature prior to being frozen at $-20^{\circ} \mathrm{C}$ until assayed. Levels of serum testosterone, oestradiol and dehydroepiandrosterone-sulfate (DHEA-s) were analysed by an electrochemiluminescence immunoassay (ECLIA, Roche) using an automated hormone analyser (Roche Elecsys, USA). Serum CT was measured by an enzymelinked immunosorbent assay (ELISA) (R\&D Systems, Minneapolis, MN, USA).

Cytokine assays - The serum obtained at different days after infection was separated into small plastic vials and stored frozen at $-20^{\circ} \mathrm{C}$ until needed for experiments. Serum cytokines were measured by specific two-site ELISAs according to the manufacturer's specifications. Briefly, ELISA plates coated with the corresponding anti-cytokine antibody were incubated with a previously established optimal serum dilution for $3 \mathrm{~h}$. After incubation, plates were washed and treated with an anti-cytokine antibody conjugated with peroxidase for $1 \mathrm{~h}$. Next, plates were washed and incubated with a colour-developing reagent $\left(\mathrm{H}_{2} \mathrm{O}_{2}-3,30-5,50\right.$-tetramethylbenzidine). The reaction was stopped $30 \mathrm{~min}$ later with $2 \mathrm{~N} \mathrm{H}_{2} \mathrm{SO}_{4}$. All samples were assayed in duplicate and the plates were read on an ELISA reader at $405 \mathrm{~nm}$. TNF- $\alpha$ (limit of detection $12.5 \mathrm{pg} / \mathrm{mL}$ ) and IL-10 (limit of detection $15 \mathrm{pg} / \mathrm{mL}$ ) detection kits were purchased from R\&D Systems (Minneapolis, MN, USA) and Pharmingen (San Diego, CA, USA), respectively.

Detection of specific antibodies - Individual serum was analysed using a commercial antibody capture ELISA (ChagaTest Recombinant 3.0, Wiener Laboratories, Rosario, Argentina) that was modified for the recognition of rat antibodies. Briefly, ELISA plates coated with recombinant $T$. cruzi antigens were incubated with a previously established optimal dilution of rat sera for $90 \mathrm{~min}$ at $37^{\circ} \mathrm{C}$. The plates were washed three times and incubated for another $90 \mathrm{~min}$ at $37^{\circ} \mathrm{C}$ with peroxidaseconjugated goat anti-rat $\operatorname{IgM}$ or $\operatorname{IgG}$ (whole molecules) or IgG isotypes (IgG1, IgG2a, IgG2b and IgG2c) (Sigma, St Louis, MO, USA). After $30 \mathrm{~min}$, plates were washed three times and incubated with a colour-developing reagent $\left(\mathrm{H}_{2} \mathrm{O}_{2}-3,30-5,50\right.$-tetramethylbenzidine). The reaction was stopped $30 \mathrm{~min}$ later with $2 \mathrm{~N} \mathrm{H}_{2} \mathrm{SO}_{4}$. The optical density was measured at $450 \mathrm{~nm}$.

Statistical analysis - All experiments were repeated at least twice. Data were expressed as mean \pm standard error of the mean. Statistical comparisons were made by the Kruskal-Wallis nonparametric analysis of variance and the Mann-Whitney U test. Correlation analysis was performed using the Spearman test. Differences were considered significant when $\mathrm{p}<0.05$.

Ethics - Animals were maintained according to International Guidelines for the Care and Use of Laboratory Animals. 


\section{RESULTS}

Parasitaemia and tissue parasitism throughout acute T. cruzi infection were inversely related to age - We analysed the parasitological features of young and adult infected rats. According to our previous results (Pascutti et al. 2003), parasitaemia levels in young rats were significantly higher than adults, with a peak value around day 7-10 pi and a progressive decline until the differences became unnoticeable on day 28 pi (Fig. 1A). By contrast, parasites were hardly detectable in adult rats from blood and heart tissue throughout the acute period. Young rats developed an acute myocarditis and showed mild and moderate infiltrates (Fig. 1B, C) and isolated amastigote nests (Fig. 1D). Lesions in most adult rats were either barely detectable or undetectable (images not shown). There were no differences in the relative weight of heart tissues between groups throughout infection. Although the acute phase is longer for young animals than adults, for comparative purposes, we decided to study the evolution of endocrine hormones and immune mediators until day 28 pi in both groups.

Testosterone levels displayed a reduction during the acute period of $T$. cruzi infection - Testosterone basal levels (day 0 pi) were lower in young rats compared with
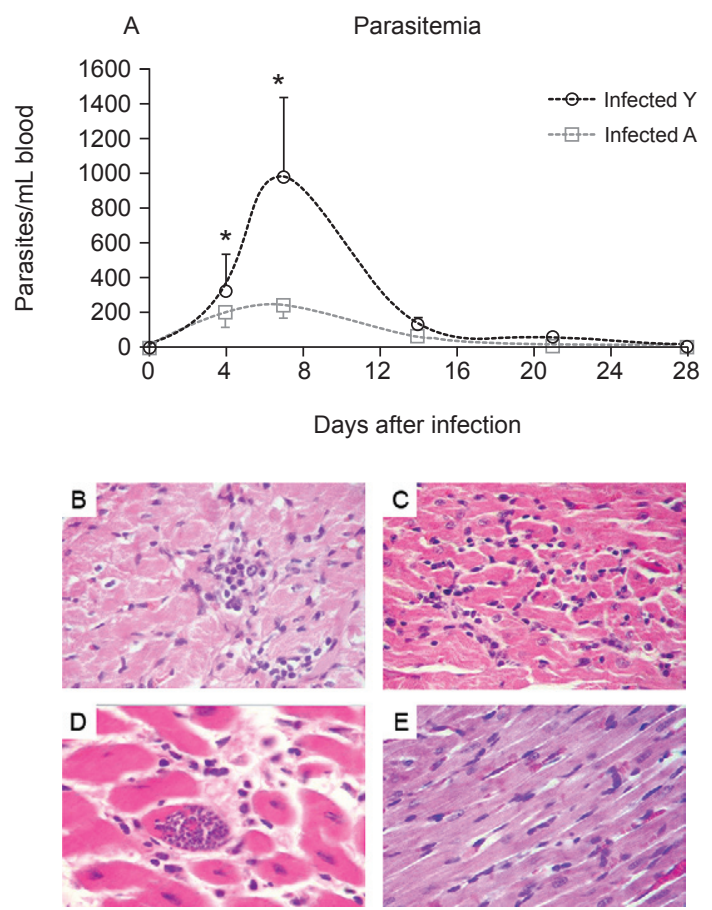

Fig. 1: parasitemia and myocarditis in acutely Trypanosoma cruzi-infected rats of different age. Parasitemia (A): data correspond to mean \pm standard error of the mean of parasites $/ \mathrm{mL}$ from eight infected young (Y) and adult (A) rats/group, inoculated by subcutaneous route. Histopathological evaluation of acute myocarditis in infected $\mathrm{Y}$ animals: mild infiltration (B); moderate infiltration (C); amastigote nest (D); normal tissue (E) (20X magnification). Five paraffin-embedded $5 \mu \mathrm{m}$ sections of 5-8 animals/day were stained with haematoxylin and eosin for evaluation of inflammatory foci and tissue parasitism. Asterisk means $\mathrm{p}<0.05$ statistically different from the infected A group. adults and they increased with reproductive maturation. At day 28 pi (when young animals were around 50-56 days old), both control groups presented similar values, which indicates that the young group had reached sexual maturity (Fig. 2A). At day seven pi, testosterone levels were significantly different between infected groups. Coincidently, young rats showed the peak in parasitaemia on that same day. After day seven pi, both groups tended to have similar hormone levels. Both infected rat groups showed lower levels of testosterone at day 21 pi compared with noninfected counterparts, but values were similar one week later (day 28 pi). In addition, histological studies in testes, seminal vesicles and epididymides did not show any differences between control and infected animals in either age group. Moreover, inflammatory infiltrate and amastigote nests were scarce or undetectable in these tissues. No differences were observed in the relative weights of testes, seminal vesicles and epididymides between infected young and adult rats (data not shown).

Oestradiol levels increased during the acute period independently of the host's age - Compared with females, lower amounts of oestradiol are naturally produced in males by testes and, to some extent, the adrenal cortex. Because oestradiol levels may be altered during an infectious process and a slight hormone change in male rats could influence immune response (particularly B-
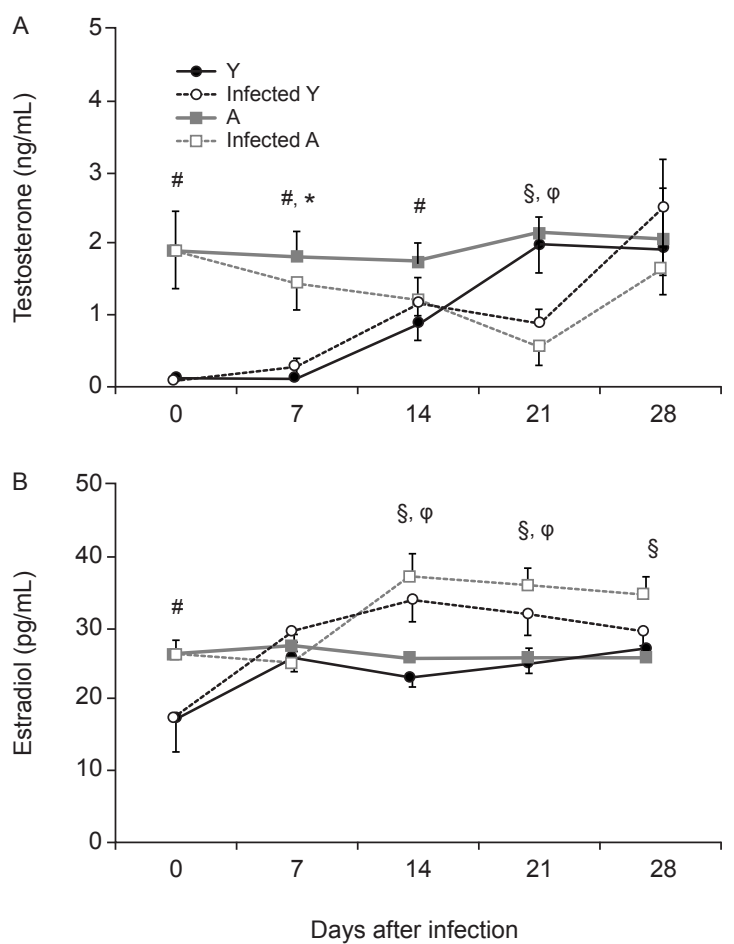

Fig. 2: serum levels of sexual hormones throughout acute Trypanosoma cruzi. Infection (A), serum testosterone and (B) estradiol levels from young (Y) and adult (A) rats in control and T. cruzi-infected groups throughout acute period. Values represent mean \pm standard error of the mean of 6-9 rats/group/day. \#: $\mathrm{p}<0.05=\mathrm{Y}$ vs. $\mathrm{A} ; \xi$ : $\mathrm{p}<$ $0.05=\mathrm{A}$ vs. infected A; $\varphi$ p: $<0.05=\mathrm{Y}$ vs. infected $\mathrm{Y}$. *: $\mathrm{p}<0.05=$ infected $\mathrm{Y}$ vs. infected $\mathrm{A}$. 
cell response facilitation), we investigated oestradiol production. At the onset of infection, young animals had significantly less oestradiol than adults, but these values were similar one week later. Remarkably, both infected groups showed enhanced levels of oestradiol compared with control counterparts: in the younger group, oestradiol was significantly increased from day 14-21 pi whereas, in adults, oestradiol was significantly increased from day 14 to the end of the experiment. Nevertheless, oestradiol concentrations were similar between infected groups during these same periods (Fig. 2B).

DHEA-s levels were unaffected whereas CT levels were higher in young compared with adult infected rats - At the beginning of the experiment, DHEA-s was higher in young rats compared with adult rats, but there was a noticeable turn down over the course of the study in the younger group. Indeed, differences in serum levels of DHEA-s among infected vs. noninfected counterparts or between infected groups were not statistically significant (Fig. 3A).

CT did not differ between noninfected groups throughout the experimental period, but infected young animals had higher $\mathrm{CT}$ levels than their adult counterparts (Fig. 3B). Indeed, the levels at days 14-21 were almost twice as high as initial levels and infected young

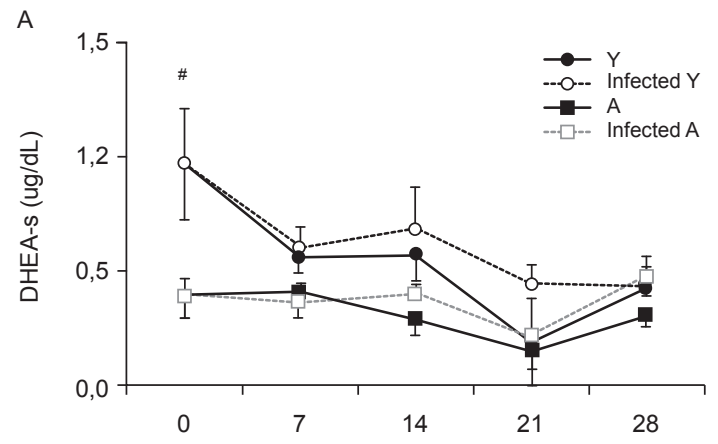

B

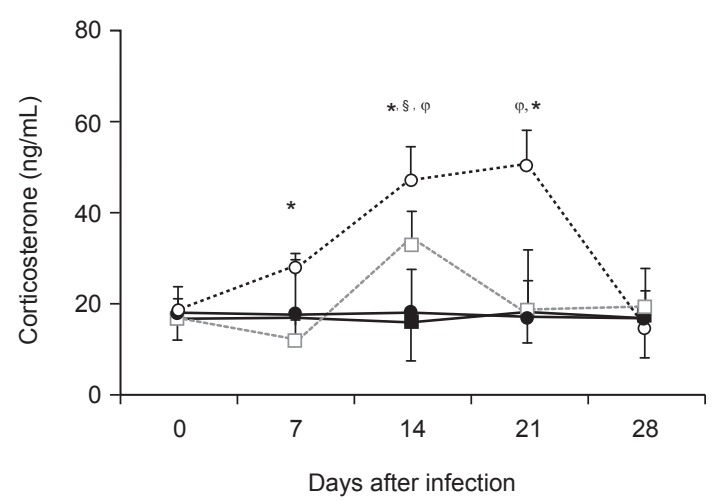

Fig. 3: serum levels of adrenal hormones throughout acute Trypanosoma cruzi. Infection (A), serum dehydroepiandrosterone-sulfate (DHEA-s) and (B) corticosterone levels from young (Y) and adult (A) rats in control and T. cruzi-infected groups throughout post-infection period. Values represent mean \pm standard error of the mean of eight rats/group/day. \#: $\mathrm{p}<0.05 \mathrm{Y}$ vs. A; $\S: \mathrm{p}<0.05 \mathrm{~A}$ vs. infected A; $\varphi$ : Y vs. infected $\mathrm{Y} ;{ }^{*}: \mathrm{p}<0.05$ infected $\mathrm{Y}$ vs. infected A. animals developed adrenal hypertrophy [relative adrenal weight ( $\mathrm{g} / \mathrm{g} \times 100)$, control young rats: $2.45 \pm 0.20$, infected young rats: $3.45 \pm 0.26, \mathrm{n}=6$-8/group, $\mathrm{p}=0.014]$. The relative adrenal weight remained constant in adult rats after infection.

Hormonal levels were affected in an age-dependent manner in response to T. cruzi infection. Because an adequate balance among hormones might be essential for appropriate development of the immune response during the infectious process, gonadal and adrenal axes were analysed.

Gonadal axis: testosteroneloestradiol (T/E $E_{2}$ ratio - Androgens are known to inhibit cellular immune responses whereas oestrogens enhance humoural immune activity, which suggests that a proper $\mathrm{T} / \mathrm{E}_{2}$ hormonal balance is likely to be important for efficient immune function. At the onset of the experiment, the $\mathrm{T} / \mathrm{E}_{2}$ ratio in noninfected young rats was lower than in adults, but the ratios of each group reached similar values after 21 days, which indicates that the young rats had reached sexual maturity (Fig. 4A). Infection modified the $\mathrm{T} / \mathrm{E}_{2}$ ratio. Indeed, at 21 days pi, the ratio began to decline in young and adult rats and the ratio reached basal levels by the end of the experiment. The $\mathrm{T} / \mathrm{E}_{2}$ ratio between infected groups, however, was similar during this period.

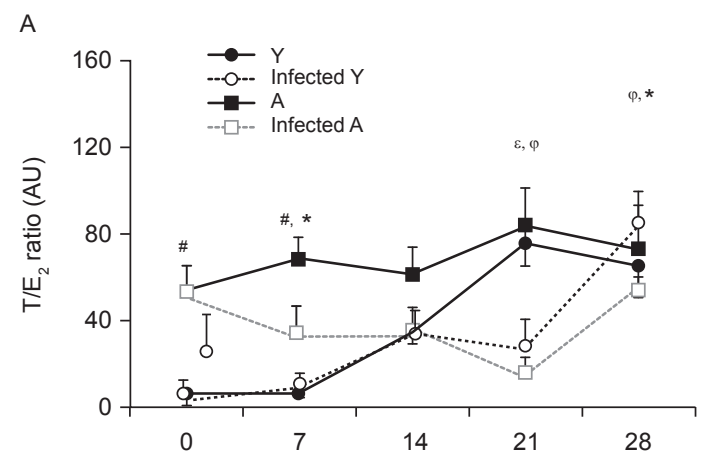

B

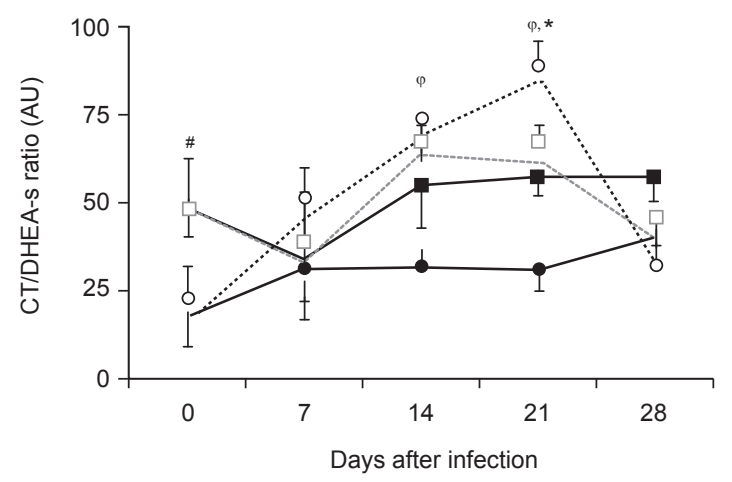

Fig. 4: serum hormonal ratios: A: testosterone/estradiol ratio $\left(\mathrm{T} / \mathrm{E}_{2}\right)$; B: corticosterone/dehydroepiandrosterone-sulfate (CT/DHEA-s) ratio from young (Y) and adult (A) rats in controls and infected groups throughout post-infection period. Values are represented as arbitrary units (AU) (mean \pm standard error of the mean) of 6-9 rats/group/day. $\#: p<0.05$ Y vs. A; $\S: p<0.05$ A vs. infected A; $\varphi$ : Y vs. infected $Y ;$ *: $\mathrm{p}<0.05$ infected $\mathrm{Y}$ vs. infected A. 
Hypothalamic-pituitary-adrenal (HPA) axis: CT/ DHEA-s ratio - Some studies have suggested that cytokine release (in situ or in circulation) may alter the CT/DHEA-s ratio and an increased CT/DHEA-s ratio has been associated with enhanced susceptibility to intracellular pathogens. Furthermore, a preserved or diminished CT/DHEA-s ratio seems to support a Th1 shift in the immune response, which favours host resistance. Although the CT/DHEA-s ratio was higher in adult rats compared with young rats at the beginning of the experiment, adult rats not showed significant variations throughout the experiment independent of whether they were infected (Fig. 4B). In contrast, young infected animals exhibited higher values compared with their control counterparts between 14-21 days pi, which reduced the defence against infection. Consistent with these observations, the CT/DHEA-s ratio was significantly higher in young infected rats compared with their infected adult counterparts.

TNF- $a$ and IL-10 levels are different between young and adult animals - Production of adrenal and gonadal steroids might be strongly influenced by inflammatory cytokines and the ensuing systemic repercussion. We analysed TNF- $\alpha$ because it is vital in the response against $T$. cruzi, HPA axis activation and testicular function. We also studied IL-10 as a major TNF- $\alpha$ counterregulatory cytokine.

Table shows that TNF- $\alpha$ levels were higher in young rats until the end of the second week pi, becoming undetectable from that point onwards. IL-10 levels were elevated in adult rats at day seven pi, but they were similar to the young rats by day 14 pi. Interestingly, the TNF- $\alpha / \mathrm{IL}-$ 10 ratio was significantly higher in young rats compared with adults at day seven and 14 pi. No correlation was observed between cytokines and hormones at any time (data not shown); this was probably because immune and hormonal responses displayed different kinetic patterns.
Oestrogen and IgG levels are associated in adult rats - Hormones are known to affect the humoural response: oestrogens increase the B-cell response whereas androgens diminish antibody production. In addition, glucocorticoids favour Th2-mediated antibody production. This evidence, along with the observation of protective antibody production in adult rats vs. the young group (Pascutti et al. 2003), prompted us to analyse whether there was an association between the steroid response and anti $T$. cruzi antibody production. As expected, IgG exhibited the same pattern as previously observed (Fig. $5 \mathrm{~A})$, whereas IgM antibody production diminished until day 28 pi (data not shown). We also studied the kinetics of IgG subclass production during the acute infection period. In line with the data on total IgG, specific IgG1, $\operatorname{IgG} 2 \mathrm{a}, \operatorname{IgG} 2 \mathrm{~b}$ and $\operatorname{IgG} 2 \mathrm{c}$ appeared earlier and showed higher amounts in the adult group than in young animals. Fig. 5A shows the evolution of the IgG2b subclass with time and indicates a protective profile in our rat model (Didoli et al. 2000). Data on other subclasses followed a similar pattern of variation (data not shown).

We also performed correlation analyses between hormone concentrations or their ratios and specific IgM, total $\mathrm{IgG}$ and $\operatorname{IgG}$ subtype levels at each time point to establish a potential association between endocrine profiles and the anti T. cruzi humoural response. Fig. 5B shows that oestrogen and specific total IgG levels were positively correlated at day seven pi in adult rats $(\mathrm{n}=$ $8 ; \mathrm{rs}=0.809 ; \mathrm{p}<0.05)$. Pairwise correlations between other hormones or hormone ratios with specific antibodies were not statistically significant (data not shown).

\section{DISCUSSION}

The immune response to parasitic infection has been shown to be paralleled by an endocrine response and gonadal and adrenal hormones are capable of modulating the immune process (Morales-Montor \& Hall 2007, Pérez

TABLE

Cytokine levels and ratio

\begin{tabular}{|c|c|c|c|c|c|}
\hline \multirow[b]{2}{*}{ Cytokines and their ratio } & \multicolumn{5}{|c|}{ Days after infection } \\
\hline & 0 & 7 & 14 & 21 & 28 \\
\hline \multicolumn{6}{|l|}{ TNF- $\alpha(p g / m L)$} \\
\hline Infected Y & ND & $24.9 \pm 4.3^{a}$ & $13.2 \pm 2.9^{a}$ & ND & ND \\
\hline Infected A & ND & $15.2 \pm 3.2$ & ND & ND & ND \\
\hline \multicolumn{6}{|l|}{ IL-10 (pg/mL) } \\
\hline Infected Y & ND & $8.5 \pm 1.4^{a}$ & $7.4 \pm 1.5$ & $1.7 \pm 0.4$ & ND \\
\hline Infected A & ND & $29.8 \pm 3.1$ & $3.1 \pm 1.0$ & $2.1 \pm 0.6$ & ND \\
\hline \multicolumn{6}{|l|}{ TNF- $\alpha /$ IL-10 } \\
\hline Infected Y & - & $2.9 \pm 0.5^{a}$ & $1.8 \pm 0.6$ & 0 & - \\
\hline Infected A & - & $0.5 \pm 0.1$ & 0 & 0 & - \\
\hline
\end{tabular}

$a: \mathrm{p}<0.05$ statistically different from the infected A. Data represents amounts of cytokines or cytokine ratio from 6-8 rats/group/ day (mean \pm standard error of the mean). Detectable interleukin (IL)-10 levels with non-detectable tumor necrosis factor $\alpha$ (TNF- $\alpha$ ) levels are indicated as a ratio zero. A: infected adults animals; ND: not determined; Y: infected young animals. 
A

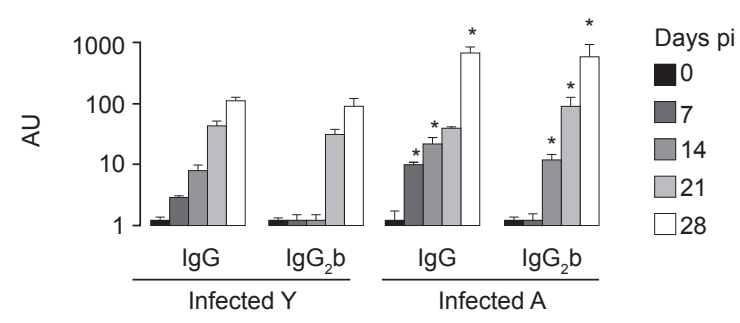

B

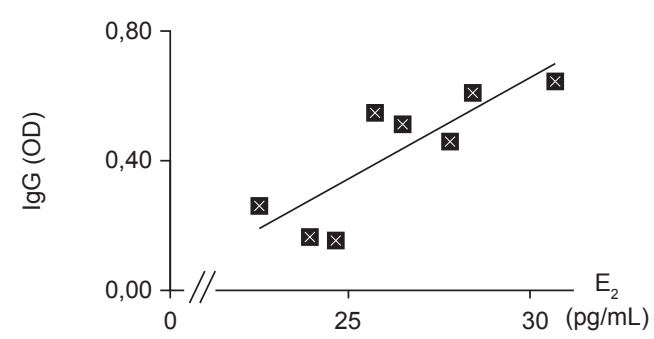

Fig. 5: antibody levels and correlations with hormones: A: total specific IgG and IgG2b levels in infected young (Y) and adult (A) animals expressed as arbitrary units (AU); B: correlations between total specific IgG and oestrogen ( $\left.\mathrm{E}_{2}\right)$ in infected A animals at seven days post-infection (pi). Optimal dilution from each serum was evaluated as optical density (OD). Correlation data represent individual values of eight rats. Spearman correlation test report an $r=0.809(p<0.05)$. Asterisk means $\mathrm{p}<0.05$ infected $\mathrm{Y}$ vs. infected $\mathrm{A}$.

et al. 2009a, Roggero et al. 2009). A study carried out in a rat model developed in our laboratory showed age associated susceptibility in the course of acute $T$. cruzi infection (Revelli et al. 1987).

In that report, inoculation of young rats resulted in a self-resolving infection that was characterised by marked parasitaemias and delayed production of specific antibodies. In adult rats, however, there were almost undetectable blood parasites and no tissue damage, even though they were inoculated with a seven-fold higher dose than young animals (Revelli et al. 1987, Pascutti et al. 2003).

Herein, we presented data consistent with the connection between age-related severity and changes in endocrine response during $T$. cruzi acute infection.

The inflammatory milieu observed in infected young animals [i.e., high TNF- $\alpha$ levels and TNF- $\alpha /$ IL-10 ratio (Table)] was adequate for parasite clearance, but this response failed to eradicate the infection and promoted more tissue damage. In addition to the immaturity of the immune system described in young rats, our results might be associated with elevated CT values and an elevated $\mathrm{CT}$ /DHEA-s ratio, which favours immunosuppression and susceptibility (Figs 3B, 4B). These results are in agreement with observations in T. cruzi-infected mice that TNF- $\alpha$ is critical for protection during acute Chagas disease, but could also be deleterious when excessively produced, which would induce vulnerability and death (Roggero et al. 2002, 2004, 2009). In addition, similar to studies in mice (Roggero et al. 2006, Pérez et al. 2007),
TNF- $\alpha$ production in our infected young rats preceded HPA axis activation and resulted in increased CT levels. The CT response observed in young rats may represent an aggravation in view of the functional alterations that glucocorticoids exert on immune cell populations (Sapolsky et al. 2000). In addition, CT increase was paralleled by a reduction in DHEA-s, which suggests that there were decreased levels of the nonsulfated DHEA. Because DHEA has been shown to protect against other protozoan infections, such as malaria (Kurtis et al. 2001) and leishmaniasis (Galindo-Sevilla et al. 2007), a decrease in DHEA could weaken the ability to fight the $T$. cruzi infection. Furthermore, Santos et al. (2008, 2010) showed that DHEA supplementation exerted stimulatory effects on the cell-mediated specific immune response during T. cruzi infection, which decreased the parasite load in blood and tissues; this suggests that DHEA-s can be used as an adjuvant for the host's immune response against infection. The results of the present study suggest that the high CT/DHEA-s ratio (Fig. 4B) could exert a negative influence on the protective response, which has been observed in other chronic infections, such as tuberculosis (Bozza et al. 2007) and human immunodeficiency virus (HIV) (Christeff et al. 1999). In schistosomiasis, the immunosuppressive effects of hydrocortisone and dexamethasone are counteracted by DHEA, which suggests that there is a tightly controlled balance in the secretion of these hormones that regulates the inflammatory response (Morales-Montor \& Hall 2007).

Studies carried out in adult animals have suggested a relationship between testosterone and T. cruzi susceptibility because males were more vulnerable to the infection than females and gonadectomy was associated with a better cellular immune response and enhanced protection (do Prado et al. 1999). Paradoxically, our study showed an association between low testosterone levels and enhanced parasitaemias in young rats and high testosterone concentrations in the adult group were not related with increased susceptibility (Fig. 2A). Schuster and Schaub (2001) reported that high testosterone levels associated with low CT concentrations could favour the control of T. cruzi infection and may partially explain the enhanced parasite control that was observed in adult animals. Conversely, Pinto et al. (2010) showed that orchiectomised mice displayed improved T-cell mediated immunity after infection. The present results differ from those of Pinto et al. (2010) and could be a consequence of age-related T-cell immaturity in young animals, which would favour a higher parasite burden, rather than being an influence of testosterone. These conflicting results suggest that other parameters should be considered.

Resistance to infection in adult rats is associated with an immunoendocrine scenario compatible with a protective and compensated state. In this regard, the observed increased amounts of IL-10 may regulate the inflammatory reaction and promote enhanced antibody production. Moreover, parasitaemia and TNF- $\alpha$ peaks preceded the decline in testosterone. This testosterone diminution may also contribute to a better control of a subclinical infection because depletion of testosterone in adult male animals favours anti-T. cruzi resistance (do Prado et al. 
1999, Pinto et al. 2010). Similar scenarios of testosterone hypogonadism have been identified in African trypanosomiasis (Hublart et al. 1990, Petzke et al. 1996). The reduction in testosterone values obtained in our model during the acute phase of infection may be related with an aromatisation of androgens. In line with other reports, this aromatisation could promote increased oestrogen levels (Spratt et al. 2006), which would further restrict T. cruzi growth (do Prado et al. 1998). The present data (Figs 2B, 4A, 5B) concerning high levels of oestrogen accompanied by a low $\mathrm{T} / \mathrm{E}_{2}$ ratio in adult rats fit well with an endocrine adaptive condition that favours an effective cellular and humoral immune response (Wilder \& Elenkov 1999, Elenkov et al. 2000, Bird et al. 2008).

In conclusion, the results of the current study suggest that cytokines and disease-related hormonal variations are associated with an age-dependent response in host resistance to $T$. cruzi. In the case of young rats, susceptibility seems to be linked with an immunoendocrine pattern that is driven by a high CT/DHEA-s ratio, which orchestrates a less efficient anti-infectious response. Interestingly, adult rats exhibited a better adaptive mechanism compared with young rats, which was characterised by high oestrogen levels and improved antibody production (high IgG synthesis), particularly during early infection.

\section{ACKNOWLEDGEMENTS}

To Dr Oscar Bottasso, for kindly reviewing the manuscript, to Analia Nocito and Vanina Tartalini, for her technical assistance, and to Gustavo Capriotti, from Wiener Laboratories, for kindly providing ChagaTest Recombinant 3.0 adapted for recognition of rat antibodies.

\section{REFERENCES}

Besedovsky HO, del Rey A 1996. Immuno-neuro-endocrine interactions: facts and hypotheses. Endocr Rev 17: 64-102.

Bird MD, Karavitis J, Kovacs EJ 2008. Sex differences and estrogen modulation of the cellular immune response after injury. Cell Immunol 252: 57-67.

Bozza VV, D’Attilio L, Mahuad CV, Giri AA, del Rey A, Besedovsky H, Bottasso O, Bay M L 2007. Altered cortisol/DHEA ratio in tuberculosis patients and its relationship with abnormalities in the mycobacterial-driven cytokine production by peripheral blood mononuclear cells. Scand J Immunol 66: 97-103.

Christeff N, Melchior JC, Mammes O, Gherbi N, Dalle MT, Nunez EA 1999. Correlation between increased cortisol: DHEA ratio and malnutrition in HIV-positive men. Nutrition 15: 534-539.

Corrêa-de-Santana E, Paez-Pereda M, Theodoropoulou M, Nihei OK, Gruebler Y, Bozza M, Arzt E, Villa-Verde DM, Renner U, Stalla J, Stalla G, Savino W 2006. Hypothalamus-pituitary-adrenal axis during Trypanosoma cruzi acute infection in mice. J Neuroimmunol 173: 12-22.

del Vecchio Filipin M, Caetano LC, Brazão V, Santello FH, Toldo MP, do Prado JC Jr 2010. DHEA and testosterone therapies in Trypanosoma cruzi-infected rats are associated with thymic changes. Res Vet Sci 89: 98-103.

Didoli GL, Davila HO, Feldman S, di Masso R, Revelli SS, Bottasso OA 2000. Protected Trypanosoma cruzi infection in rats born to mothers receiving interferon-gamma during gestation is associated with a decreased intramacrophage parasite growth and preferential synthesis of specific IgG2b antibodies. Int J Immunopharmacol 22: 45-55. do Prado Jr JC, Leal M de P, Anselmo-Franci JA, de Andrade HF Jr, Kloetzel JK 1998. Influence of female gonadal hormones on the parasitemia of female Calomys callosus infected with the "Y" strain of Trypanosoma cruzi. Parasitol Res 84: 100-105.

do Prado Jr JC, Levy AM, Leal MP, Bernard E, Kloetzel JK 1999. Influence of male gonadal hormones on the parasitemia and humoral response of male Calomys callosus infected with the Y strain of Trypanosoma cruzi. Parasitol Res 85: 826-829.

Elenkov IJ, Chrousos GP, Wilder RL 2000. Neuroendocrine regulation of IL-12 and TNF-alpha/IL-10 balance. Clinical implications. Ann N Y Acad Sci 917: 94-105.

Galindo-Sevilla N, Soto N, Mancilla J, Cerbulo A, Zambrano E, Chavira R, Huerto J 2007. Low serum levels of dehydroepiandrosterone and cortisol in human diffuse cutaneous leishmaniasis by Leishmania mexicana. Am J Trop Med Hyg 76: 566-572.

Hublart M, Tetaert D, Croix D, Boutignon F, Degand P, Boersma A 1990. Gonadotropic dysfunction produced by Trypanosoma brucei brucei in the rat. Acta Trop 47: 177-184.

Kurtis J, Mtalib R, Onyango FK, Duffy PE 2001. Human resistance to Plasmodium falciparum increases during puberty and is predicted by dehydroepiandrosterone sulfate levels. Infect Immun 69: 123-128.

Morales-Montor J, Hall CA 2007. The host-parasite neuroimmunoendocrine network in schistosomiasis: consequences to the host and the parasite. Parasite Immunol 29: 599-608.

Nakazawa M, Fantappie MR, Freeman GL Jr, Eloi-Santos S, Olsen NJ, Kovacs WJ, Secor WE, Colley DG 1997. Schistosoma mansoni: susceptibility differences between male and female mice can be mediated by testosterone during early infection. Exp Parasitol 85: 233-240.

Pascutti MF, Bottasso OA, Hourquescos MC, Wietzerbin J, Revelli S 2003. Age-related increase in resistance to acute Trypanosoma cruzi infection in rats is associated with an appropriate antibody response. Scand J Immunol 58: 173-179.

Pérez AR, Bottasso OA, Savino W 2009a. The impact of infectious diseases upon neuroendocrine circuits. Neuroimmunomodulation 16: 96-105.

Pérez AR, Fontanella GH, Nocito AL, Revelli S, Bottasso OA 2009b. Short treatment with the tumour necrosis factor-alpha blocker infliximab diminishes chronic chagasic myocarditis in rats without evidence of Trypanosoma cruzi reactivation. Clin Exp Immunol 157: 291-299.

Pérez AR, Roggero E, Nicora A, Palazzi J, Besedovsky HO, del Rey A, Bottasso OA 2007. Thymus atrophy during Trypanosoma cru$z i$ infection is caused by an immuno-endocrine imbalance. Brain Behav Immun 7: 890-900.

Petzke F, Heppner C, Mbulamberi D, Winkelmann W, Chrousos GP, Allolio B, Reincke M 1996. Hypogonadism in Rhodesian sleeping sickness: evidence for acute and chronic dysfunction of the hypothalamic-pituitary-gonadal axis. Fertil Steril 65: 68-75.

Pinto AC, Caetano LC, Levy AM, Fernandes RD, Santos CD, do Prado JC Jr 2010. Experimental Chagas' disease in orchiectomized Calomys callosus infected with the CM strain of Trypanosoma cruzi. Exp Parasitol 124: 147-152.

Revelli S, Moreno H, Berra H, Valenti JL, Nocito AL, Amerio N, Morini JC 1987. Influencia de la edad de la rata en la evolución de la infección con Trypanosoma cruzi. Medicina 47: 360-366.

Roberts CW, Walker W, Alexander J 2001. Sex-associated hormones and immunity to protozoan parasites. Clin Microbiol Rev 3: 476-488.

Roggero E, Pérez A, Tamae-Kakazu M, Piazzon I, Nepomnaschy I, Wietzerbin J, Serra E, Revelli S, Bottasso O 2002. Differential susceptibility to acute Trypanosoma cruzi infection in BALB/c 
and C57BL/6 mice is not associated with a distinct parasite load but cytokine abnormalities. Clin Exp Immunol 128: 421-428.

Roggero E, Pérez AR, Bottasso OA, Besedovsky HO, del Rey A 2009. Neuroendocrine-immunology of experimental Chagas' disease. Ann NY Acad Sci 1153: 264-271.

Roggero E, Pérez AR, Tamae-Kakazu M, Piazzon I, Nepomnaschy I, Besedovsky HO, Bottasso OA, del Rey A 2006. Endogenous glucocorticoids cause thymus atrophy but are protective during acute Trypanosoma cruzi infection. J Endocrinol 190: 495-503.

Roggero E, Piazzon I, Nepomnaschy I, Pérez A, Velikovsky A, Revelli S, Bottasso O 2004. Thymocyte depletion during acute Trypanosoma cruzi infection in C57BL/6 mice is partly reverted by lipopolysaccharide pre-treatment. FEMS Immunol Med Microbiol 41: 123-131.

Santos CD, Loria RM, Oliveira LG, Kuehn CC, Toldo MP, Albuquerque S, do Prado JC Jr 2010. Effects of dehydroepiandrosteronesulfate (DHEA-S) and benznidazole treatments during acute infection of two different Trypanosoma cruzi strains. Immunobiology 215: 980-986.

Santos CD, Toldo MP, Santello FH, Filipin M del V, Brazão V, do Prado JC Jr 2008. Dehydroepiandrosterone increases resistance to experimental infection by Trypanosoma cruzi. Vet Parasitol 153: 238-243.
Sapolsky RM, Romero LM, Munck AU 2000. How do glucocorticoids influence stress responses? Integrating permissive, suppressive, stimulatory and preparative actions. Endocr Rev 21: 55-89.

Schuster JP, Schaub GA 2001. Experimental Chagas disease: the influence of sex and psychoneuroimmunological factors. Parasitol Res 12: 994-1000.

Spratt DI, Morton JR, Kramer RS, Mayo SW, Longcope C, Vary CP 2006. Increases in serum estrogen levels during major illness are caused by increased peripheral aromatization. Am J Physiol Endocrinol Metab 291: 631-638.

Storino RA, Milei J 1994. Enfermedad de Chagas, Mosby Doyma, Buenos Aires, 209 pp.

Wilder RL, Elenkov IJ 1999. Hormonal regulation of tumor necrosis factor-alpha, interleukin-12 and interleukin-10 production by activated macrophages. A disease-modifying mechanism in rheumatoid arthritis and systemic lupus erythematosus. Ann NY Acad Sci 876: 14-31.

WHO/PAHO - World Health Organization/Pan American Health Organization Regional Office 2007. The burden of neglected diseases in Latin America and the Caribbean compared with some other communicable diseases. Available from: www.paho.org/ English/AD/DPC/CD/psit-nd-graph.htm. 